\title{
CRITICAL CONDITIONS OF IGNITION OF FUEL SPRAY CONTAINING LIQUID FUEL DROPLETS
}

\author{
A.Zh. Agataeva, E.A. Shchepakina \\ ${ }^{1}$ Samara National Research University, Samara, Russia
}

\begin{abstract}
The ignition of combustible gas containing liquid fuel droplets is investigated. The analysis is based on the theory of integral manifolds of singularly perturbed systems. This approach allows us to define different types of chemical regimes including the critical mode. The relation between the critical regime and the phenomenon of delayed loss of stability in the dynamical model is shown.
\end{abstract}

Keywords: integral manifold, singular perturbations, delayed loss of stability, spray, combustion theory, critical behavior, thermal explosion.

Citation: Agataeva AZh, Shchepakina EA. Critical conditions of ignition of fuel spray containing liquid fuel droplets. CEUR Workshop Proceedings 2016; 1638: 484-492. DOI: 10.18287/1613-0073-2016-1638-484-492

\section{Introduction}

In this paper we present a qualitative investigation of combustion dynamics in a multiphase medium. The investigation of the ignition process in a gas medium was carried out by many authors, see, for instance, [1-16] and references therein. However, the influence of liquid droplets within such a context is less studied [17]. The dynamics of such systems is determined by two processes: heat loss due to the evaporation of the flammable liquid medium (droplets) and heat release associated with an exothermic oxidation reaction in the gas phase $[17,18]$. Competition between these processes determines the main dynamical features of the systems.

\section{Model}

A concise physical model for the thermal explosion in a two-phase medium (combustible gas mixture - combustible liquid drops) is suggested using an adiabatic approach. The main physical assumptions of the model are as follows. The combustible liquid droplets are considered to comprise a monodisperse spray, whose effect on the blowup process is to be investigated qualitatively. As usual [2] for thermal explosion processes, we neglect the pressure change in the reaction volume and its influence on the combustion process. We assume that the thermal conductivity of the liquid phase is much greater than that of the gas phase. Thus, the heat transfer coefficient in the liq- 
uid gas mixture is supposed to be defined by the thermal properties of the gas phase. It is assumed that the quasi-steady state approximation is valid for the vaporizing droplets [18]. The drop boundary is assumed to be on a saturation line (i.e., the liquid temperature is constant and is equal to the liquid saturation temperature). The combustion reaction is modeled as a first order, highly exothermic chemical reaction. The exothermic oxidation reaction is usually modelled as a single step reaction obeying an Arrhenius temperature dependence. Heat losses were assumed proportional to the temperature excess over the ambient temperature (Newtonian cooling) [1, 4].

Under these assumptions a mathematical model of the process has a form [18]:

$c_{p g} \rho_{g} \alpha_{g} \frac{d T_{g}}{d t}=C_{f} C_{o x} Q_{f} \alpha_{g} Z \mu_{f} \exp \left(-\frac{E}{R T_{g}}\right)-4 \pi R_{d} \lambda_{g} n_{d}\left(T_{g}-T_{d}\right)$,

$\frac{d\left(R_{d}^{3}\right)}{d t}=\frac{3 \lambda_{g}}{\rho_{L} R_{d} L}\left(T_{d}-T_{g}\right)$,

$\frac{d C_{f}}{d t}=-C_{f} C_{o x} Z \exp \left(-\frac{E}{R T_{g}}\right)+\frac{4 \pi R_{d} \lambda_{g} n_{d}}{L \mu_{f} \alpha_{g}}\left(T_{g}-T_{d}\right)$,

$\frac{d C_{o x}}{d t}=-C_{f} C_{o x} Z \exp \left(-\frac{E}{R T_{g}}\right)$,

$\rho_{g} T_{g}=\rho_{g 0} T_{g 0}$,

where $T$ is a temperature; $R_{d}$ is a radius of the drops; $C$ is a concentration; $Z$ is preexponential factor; $E$ is an activation energy; $Q$ is a energy of the combustion; $L$ is a liquid evaporation energy; $R$ is the universal gas constant; $c$ is a thermal capacity; $n_{d}$ is a number of drops per unit volume; $t$ is a time; $\alpha$ is a volumetric phase content; $\lambda$ is a thermal conductivity; $\rho$ is a density; $\mu$ is a molar mass. The subscripts here denote: $d$ - liquid fuel droplets; $f$ - combustible gas component of the mixture; $g$ - gas mixture; $L$ - liquid; $p$ - under constant pressure; $o x$ - oxidizer; 0 - initial state.

Suppose that the fuel is a deficient reagent in a large amount of oxidant, thus equation (4) can be omitted.

Following to the classical theory Semenov [1] we define the dimensionless variables

$$
\begin{aligned}
& \eta=\frac{C_{f}}{C_{f 0}}, \quad \theta=\frac{E}{R T_{g 0}} \cdot \frac{T_{g}-T_{g 0}}{T_{g 0}}, \quad r=\frac{R_{d}}{R_{d 0}}, \\
& \tau=\frac{t}{t_{\text {react }}}, \quad t_{\text {react }}=A^{-1} \exp \left(\frac{E}{R T_{g 0}}\right), \\
& \beta=\frac{R T_{g 0}}{E}, \quad \gamma=\frac{c_{p g} T_{g 0} \rho_{g 0}}{C_{f 0} Q_{f} \mu_{f}} \beta, \quad \varepsilon_{1}=\frac{4 \pi R_{d 0} \lambda_{g} \beta T_{g 0} n_{d}}{A C_{f 0} Q_{f} \alpha_{g} \mu_{f}} \exp \left(\frac{E}{R T_{g 0}}\right), \\
& \varepsilon_{2}=\frac{Q_{f} C_{f 0} \alpha_{g} \mu_{f}}{\rho_{L} L \alpha_{L}}, \quad \psi=\frac{Q_{f}}{L},
\end{aligned}
$$

and the system (1)-( 3 ) can be rewritten in dimensionless form: 
$\gamma \frac{d \theta}{d \tau}=\eta \exp \left(\frac{\theta}{1+\beta \theta}\right)-\varepsilon_{1} r \theta(1+\beta \theta)$

$\frac{d\left(r^{3}\right)}{d \tau}=-\varepsilon_{1} \varepsilon_{2} r \theta$

$\frac{d \eta}{d \tau}=-\eta \frac{1}{1+\beta \theta} \exp \left(\frac{\theta}{1+\beta \theta}\right)+\varepsilon_{1} \psi r \theta$

where $\theta$ is the dimensionless fuel gas temperature; $r$ is the dimensionless radius of the drops; $\eta$ is the dimensionless concentration of flammable gas; $\tau$ is the dimensionless time; $\gamma$ is the dimensionless parameter equal to the final dimensionless adiabatic temperature thermally isolated system after the explosion; $\beta$ gives the initial temperature; $\varepsilon_{1}, \varepsilon_{2}$ characterize the interaction between the gas and liquid phases; $\psi$ is a parameter characterizing the ratio of the energy of combustion gas mixture to the liquid evaporation energy.

The initial conditions for the equations (6)-(8) are:

$\theta(0)=0, \eta(0)=1, r(0)=1$.

It should be noted that the system (6)-(8) has the energy integral:

$\eta-1+\frac{\gamma}{\beta} \ln (1+\beta \theta)+\frac{\psi-1}{\varepsilon_{2}}\left(r^{3}-1\right)=0$.

Taking into account (9) we can reduced the system (6)-(8) [19-23]:

$\gamma \frac{d \theta}{d \tau}=\left[1-\frac{\gamma}{\beta} \ln (1+\beta \theta)-\frac{\psi-1}{\varepsilon_{2}}\left(r^{3}-1\right)\right] \exp \left(\frac{\theta}{1+\beta \theta}\right)-\varepsilon_{1} r \theta(1+\beta \theta)$,

$\frac{d\left(r^{3}\right)}{d \tau}=-\varepsilon_{1} \varepsilon_{2} r \theta$

Thus, the dynamic of the system depends on five dimensionless parameters:

$\beta \ll 1, \gamma \ll 1, \varepsilon_{1}, \varepsilon_{2}, \Psi$. The property values of $\gamma$ and $\beta$ are small compared to unity for most gas mixtures because of the high activation energy and the exothermic chemical reaction $[3,4,6,17]$. The smallness of the parameter $\gamma$ implies that (10), (11) is a singularly perturbed system, which allows us to apply the geometric methods of the singular perturbation theory for its analysis [19-23].

\section{Analysis}

The degenerate equation $[21,23]$

$\Lambda(\theta, r)=\left(1-\frac{\gamma}{\beta} \ln (1+\beta \theta)+\frac{(\psi-1)\left(1-r^{3}\right)}{\varepsilon_{2}}\right) \exp \left(\frac{\theta}{1+\beta \theta}\right)-\varepsilon_{1} r \theta(1+\beta \theta)=0$ 
describes the zero-approximation of slow integral manifold of the system, a slow curve. The analysis of the slow curve allows us to determine the basic types of chemical reaction regimes depending on the values of the additional parameters of (10), (11).

For $\psi>0$ the slow curve has a shape as shown on Figure 1. The parts PT and QS of the slow curve are stable while the part TQ is unstable. Depending on the ratio of other parameters following cases are possible: thermal explosion with delay or typical thermal explosion. In the first case a trajectory of the system, starting from the initial point $\mathrm{A}$ in the basin of attraction of the stable part PT, will be attracted to it with the velocity of the fast variable (see path $\mathrm{AB}$ ) and then follows along it with the velocity of the slow variable (see path BT). After this moment the trajectory will jump from the slow integral manifold (see path TC). The thermal explosion will have happened long before the point $\mathrm{C}$ is reached because the temperature' value at the point $\mathrm{C}$ extremely high.

The typical thermal explosion occurs if the initial point is located below the basin of attraction of the stable part PT (see the trajectory DE on Figure 1).
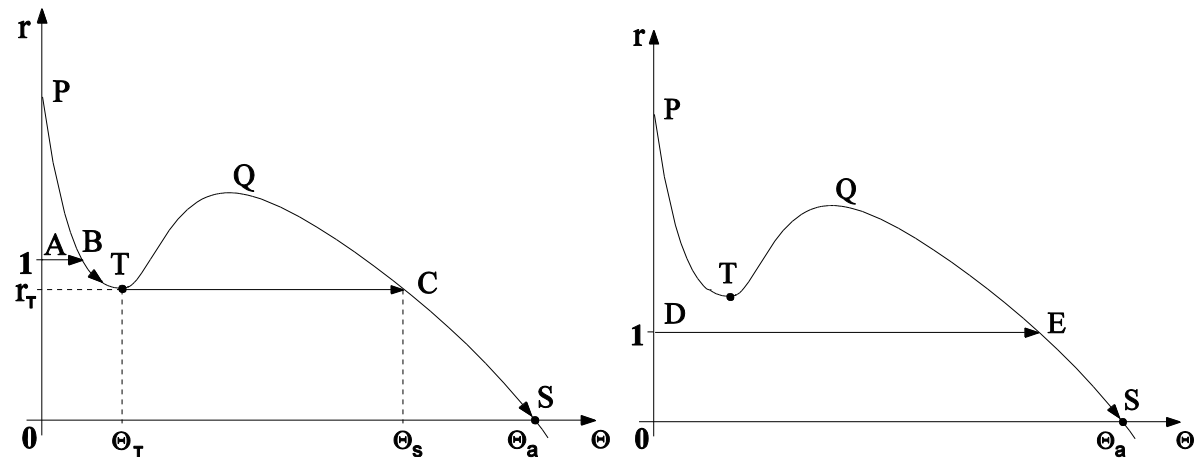

Fig. 1. Slow curve and the trajectories of (10), (11) with $\psi>0$

Figure 2 demonstrate the trajectory of the reduced system (10), (11) and the solutions of the full system (6)-(8) in the case of thermal explosion with delay for $\beta=0.05, \gamma=0.01, \varepsilon_{1}=2.0, \varepsilon_{2}=0.8, \psi=0.19$.

Figure 3 shows the slow curve and a trajectory (AB) of the system (10), (11) in the case $\psi>1$. This case also corresponds to the typical thermal explosion and the behaviors of the solutions of (6)-(8) are the similar as in previous case. 

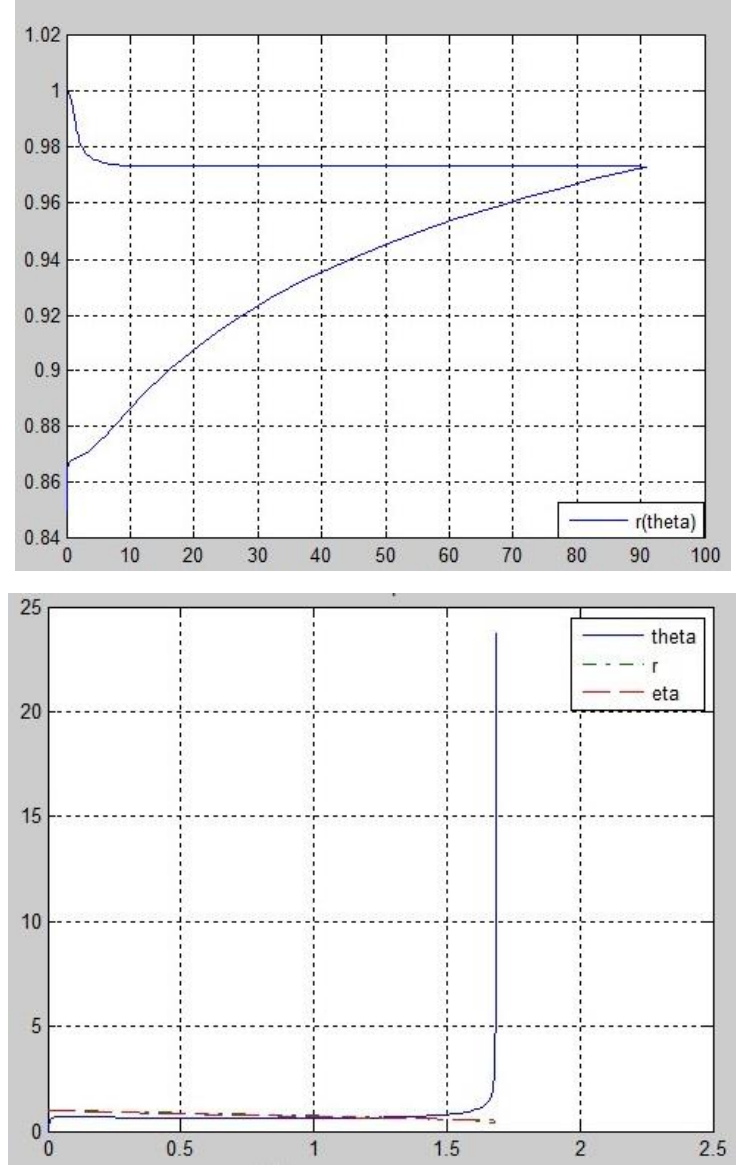

Fig. 2. Thermal explosion with delay: (top) the dimensionless radius $r$ vs. $\theta$ and (bottom) the solutions of the system (6)-(8) vs. $\tau$

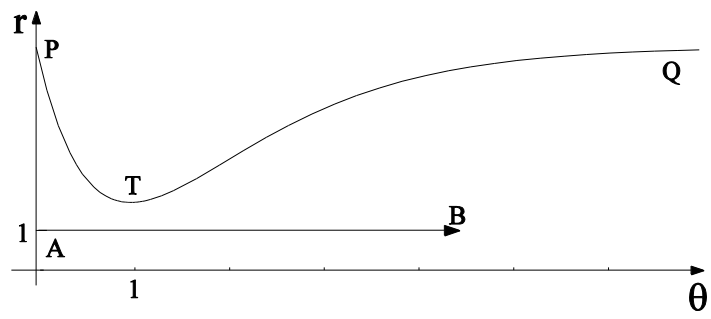

Fig. 3. Slow curve and a trajectory of the system (10), (11) with $\psi>1$

For $0<\psi<1-\varepsilon_{2}$ the slow curve is shown on Figure 4. In this case three regimes are possible depending on the ratio of the parameters: the typical thermal explosion (the trajectory $\mathrm{CD}$ ), the slow combustion regime (the trajectory CTP), and the critical regime (the trajectory CTQ). 


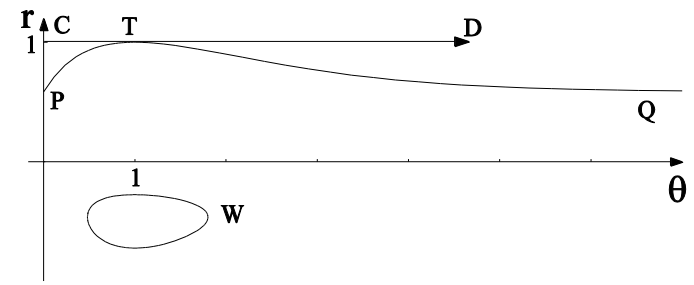

Fig. 4. Slow curve and the trajectories of the system (10), (11) with $0<\psi<1-\varepsilon_{2}$

Figure 5 demonstrate the trajectory of the system (10)-(11) and the solutions of the system (6)-(8) in the case of slow combustion regime. Without loss of generality the parameters of the system are chosen to be $\beta=0.05, \gamma=0.01, \psi=0.19, \varepsilon_{1}=3.5$, and $\varepsilon_{2}=0.8$.
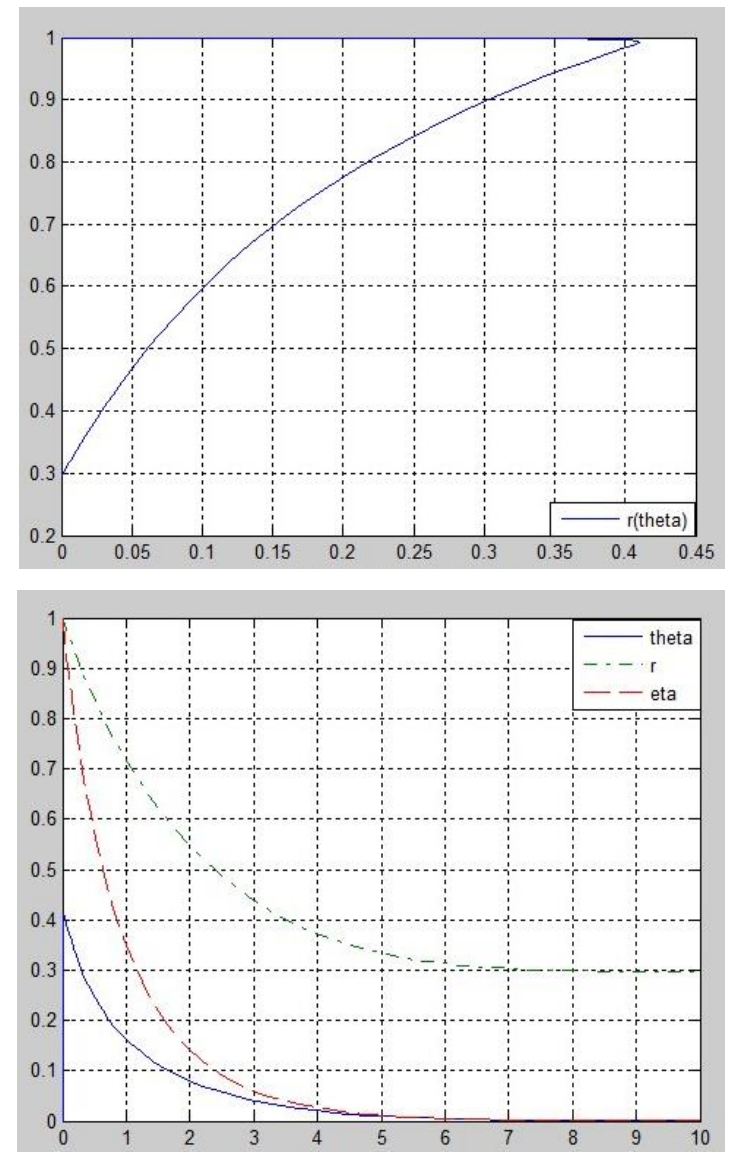

Fig. 5. Slow combustion regime: (top) the dimensionless radius $r$ vs. $\theta$ and (bottom) the solutions of the system (6)-(8) vs. $\tau$ 
The critical regime separates an explosive regime from a nonexplosive one. The crucial result is that the unstable slow manifold may be used to construct the separating regime between the safe regimes and explosive ones.

Figure 6 shows the trajectory of the system (10), (11) and the solutions of the system (6)-(8) in the case of critical regime $\left(\varepsilon_{1}=2.2\right.$, other parameters are the same as in Figure 5). The critical trajectory is characterized by a comparatively rapid (but not explosive) flow of the reaction till the essential degree of conversion takes place and then a jump slow-down and a transition to the slow flow of the reaction to near the origin.
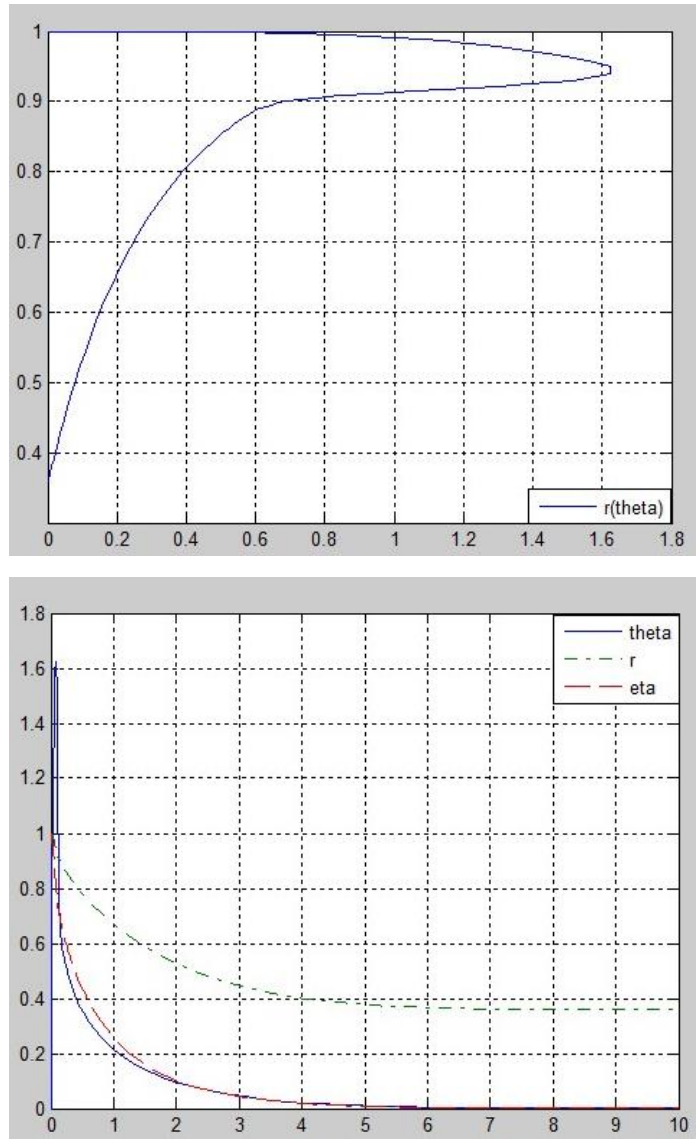

Fig. 6. Critical regime: (top) the dimensionless radius $r$ vs. $\theta$ and (bottom) the solutions of the system (6)-(8) vs. $\tau$

The critical value of a control parameter, say $\varepsilon_{1}$, corresponding to the critical trajectory may be found in the form of the asymptotic expansion [24]: 
$\varepsilon_{1}=v_{0}+v_{1} \gamma^{2 / 3}+v_{2} \gamma \ln \frac{1}{\gamma}+O(\gamma)$.

Such approach to modeling of the critical phenomena in combustion problems has been applied in [7, 12, 21, 23, 25].

\section{Conclusion}

The dynamical model of the ignition in the two-phase medium was considered. The study of the zeroth order approximation of the slow integral manifold of the system (the slow curve) has allowed to define the three basic types of chemical reaction modes: safe slow combustion mode, typical thermal explosion regime and thermal explosion regime with a delay. It was shown that the realizability conditions for these regimes depend on the values of the additional system parameters. It was shown that there is also the critical regime which divides the area of safe reactions and the area of dangerous, explosive regimes. The conditions of the existence of the critical regime have been obtained via the geometric theory of singular perturbation. The crucial result is that the unstable slow manifold may be used to construct the separating regime between the safe regimes and explosive ones.

\section{Acknowledgment}

This work is supported in part by the Russian Foundation for Basic Research (grant 14-01-97018-p) and the Ministry of Education and Science of the Russian Federation under the Competitiveness Enhancement Program of Samara University (2013-2020).

\section{References}

1. Semenov NN. Zur theorie des verbrennungsprozesses. Z. Physik. Chem., 1928; 48: 571-581. [in German]

2. Frank-Kamenetskii DA. Diffusion and Heat Exchange in Chemical Kinetics, 2nd ed. New York: Plenum Press, 1969.

3. Gray BF. Critical behaviour in chemical reacting systems: 2 . An exactly soluble model. Combust. Flame, 1973; 21: 317-325.

4. Zeldovich YaB, Barenblatt GI, Librovich VB, Makhviladze GM. The Mathematical Theory of Combustion and Explosions. New York: Consultants Bureau, 1985.

5. Gol'dshtein VM, Sobolev VA, Yablonskii GS. Relaxation self-oscillations in chemical kinetics: a model, condition for realization. Chemical Engn. Sci., 1986; 41(11): 27612766.

6. Babushok VI, Goldshtein VM, Sobolev VA. Critical condition for the thermal explosion with reactant consumption. Combust. Sci. and Tech., 1990; 70: 81-89.

7. Gorelov GN, Sobolev VA. Mathematical modelling of critical phenomena in thermal explosion thory. Combust. Flame, 1991; 87: 203-210.

8. Gorelov GN, Sobolev VA. Duck-trajectories in a thermal explosion problem. Appl. Math. Lett., 1992; 5(6): 3-6. 
9. Sobolev VA, Shchepakina EA. Self-ignition of laden medium. J. Combustion, Explosion and Shock Waves, 1993; 29(3): 378-381.

10. Sobolev VA, Shchepakina EA. Duck trajectories in a problem of combustion theory, Differential Equations, 1996; 32: 1177-1186.

11. Gol'dshtein V, Sobolev V, Zinoviev A. Thermal explosion in a dusty gas. Z. Angew. Mathem. Mech. (ZAMM), 1996; 76(S2): 533-534.

12. Gol'dshtein V, Zinoviev A, Sobolev V, Shchepakina E. Criterion for thermal explosion with reactant consumption in a dusty gas. Proc. R. Soc. Lond. A, 1996; 452: 21032119.

13. Shchepakina EA. Black swans and canards in self-ignition problem. Nonlinear Anal. Real World Appl., 2003; 4(1): 45-50.

14. Kitaeva E, Sobolev V. Numerical determination of bounded solutions to discrete singularly perturbed equations and critical combustion regimes. Computational Mathematics and Mathematical Physics, 2005; 45(1): 52-82.

15. Shchepakina E, Korotkova O. Canard explosion in chemical and optical systems. Discrete and Continuous Dynamical Systems - Series B, 2013; 18(2): 495-512.

16. Shchepakina EA. Critical phenomena in a model of fuel's heating in a porous medium. CEUR Workshop Proceedings, 2015; 1490: 179-189. DOI: 10.18287/1613-00732015-1490-179-189

17. Sazhin S. Droplets and Sprays London: Springer, 2014.

18. Goldfarb I, Gol'dshtein V, Shreiber I, Zinoviev A. Liquid drop effects on self-ignition of combustible gas. Proceedings of the 26th Symposium (International) on Combustion. Pittsburgh, The Combustion Institute, 1996; 1557-1563.

19. Kononenko LI, Sobolev VA. Asymptotic expansion of slow integral manifolds. Sib. Math. J., 1994; 35: 1119-1132.

20. Voropaeva NV, Sobolev VA. A constructive method of decomposition of singularly perturbed nonlinear differential systems. Differ. Equations, 1995; 31(4): 528-537.

21. Shchepakina E, Sobolev V. Black swans and canards in laser and combustion models. In: Mortell M, O'Malley R, Pokrovskii A, Sobolev V. (eds.) Singular Perturbations and Hysteresis. SIAM, Philadelphia, 2005; 207-256

22. Sobolev VA, Tropkina EA. Asymptotic expansions of slow invariant manifolds and reduction of chemical kinetics models. Comput. Mathematics and Math. Physics, 2012; 52(1): 75-89.

23. Shchepakina E, Sobolev V, Mortell MP. Singular Perturbations: Introduction to System Order Reduction Methods with Applications. In: Springer Lecture Notes in Mathematics, Vol. 2114. Cham: Springer International Publishing, 2014; 212 p.

24. Mishchenko EF, Rozov NKh. Differential Equations with Small Parameters and Relaxation Oscillations. New York: Plenum Press, 1980.

25. Shchepakina E, Sobolev V. Modelling of critical phenomena for ignition of metal particles. Journal of Physics: Conference Series, 2008; 138: 012025. 